\title{
CAUSAS DE MORTALIDADE NA INFÂNCIA, REGIÃO DE RIBEIRÃO PRETO, SÃO PAULO, BRASIL *
}

\author{
José Romero TERUEL ** \\ Jarbas Leite NOGUEIRA ** \\ Uilio A. GOMES **
}

RSPU-B/161

\begin{abstract}
TERUel, J. R. et al. - Causas de mortalidade na infância, região de Ribeirão Preto, São Paulo, Brasil. Rev. Saúde públ., São Paulo, 7:67-72, 1973.

Resumo: Descrição do estudo de 1124 óbitos de menores de 5 anos, residentes em áreas urbanas da região de Ribeirão Preto, no periodo de julho de 1968 a junho de 1970, através da coleta de todas as informaçóes registradas nos serviços assistenciais e de informações, obtidas em entrevistas às famílias e junto aos médicos responsáveis pelo atendimento. Foi observado o predomínio de óbitos no periodo neonatal. O coeficiente de mortalidade infantil foi estimado em 52,4 por mil nascidos vivos. Foi evidenciada a importância das causas infecciosas $e$ as perinatais, chamando a atenção para os problemas assistenciais junto à infância, principalmente a escassez de pessoal paramédico treinado.
\end{abstract}

UnITERmos: Mortalidade infantil*; Ribeirão Preto, S.P., Brasil*; Doenças infecciosas".

\section{N T R O D U Ç A O}

Os elevados coeficientes de mortalidade na infância da América Latina ${ }^{1}$ refletem a importância dos problemas de saúde que, por sua natureza, trazem repercussões aos grupos etários mais jovens.

Na VII Reunião Anual da Associação Brasileira de Escolas de Medicina 8, realizada em 1969, Araujo ${ }^{2}$, Silva $^{9}$ e SiMŌEs ${ }^{10}$, utilizando os dados disponíveis, fizeram uma apreciação sobre os problemas de saúde do Brasil, através de vá- rios indicadores. Levando em conta as grandes variações regionais, observaram que, nos anos próximos a 1964, a mortalidade infantil estava ao redor de cem óbitos por mil nascidos vivos e que a mortalidade deste grupo etário contribuia com quase $30 \%$ do total de óbitos registrados. Observaram ainda que a mortalidade em menores de 5 anos era responsável por cerca de $50 \%$ do total de óbitos.

* A investigação foi realizada no Departamento de Medicina Social da Faculdade de Medicina de Ribeirāo Preto (USP) graças a convênio com a Organização Pan-Americana da Saúde e Ministério da Saúde.

*: Do Departamento de Medicina Social da Faculdade de Medicina de Ribeirão Preto da USP - Ribelrão Preto - São Paulo, Brasil. 
TERUEL. J. R. et al. - Causas de mortalidade na infância, região de Ribeirão Preto, São Paulo, Brasil. Rev. Saúde públ., S. Paulo, 7: 67-72, 1973.

Os dados publicados pela Organização Pan-Americana da Saúde, 4,5 em 1968 e 1970 , demonstram, para os óbitos de menores de um ano, a importância das doenças próprias da primeira infância, correspondentes ao Grupo XV da 7." revisão da Classificação Internacional de Doenças. Este grupo é seguido pelas doenças infecciosas, com grande mortalidade devida às gastroenterites e pelas doenças do aparelho respiratório, principalmente as pneumonias e broncopneumonias. $\mathrm{Pa}$ ra as crianças de 1 a 4 anos são as doenças infecciosas e parasitárias que ocupam o primeiro lugar, notadamente as gastroenterites e enterocolites. O documento da Organização Pan-Americana da Saú$\mathrm{de}^{4}$ (1970) assinala que a desnutrição contribui para a morte de muitas crianças, porém, estes óbitos são atribuídos a outras afecçōes concorrentes como as doenças infecciosas, principalmente a diarréia. Conforme Araujo 2 (1969) no Brasil, as gastroenterites $e$ as doenças da primeira infância figuram como as principais causas de óbito.

No sentido de estudar os problemas de saúde na infância, a Organização PanAmericana da Saúde coordenou uma investigação, procurando analisar as causas básicas e associadas de óbito, em crianças menores de 5 anos, em 15 áreas do continente americano 7. Resultados preliminares foram publicados em 1971 pela Organização Pan-Americana da Saúde ${ }^{6}$.

Ao ser incluída a região de Ribeirão Preto, como uma das áreas selecionadas para a Investigação Interamericana de Mortalidade na Infância, foi propiciada a oportunidade de se obter dados que permitiram conhecer as causas principais dos óbitos e uma melhor estimativa dos coeficientes de mortalidade em menores de 5 anos.

\section{MATERIAL E METODOS}

Foram objeto de estudo os óbitos de crianças menores de 5 anos, cujas familias residiam na área urbana dos municípios de Ribeirão Preto, Franca, Batatais, Brodosqui, Cravinhos, Jardinópolis e Sertãozinho. As cinco últimas áreas serão designadas de "cinco comunidades" e analisadas em conjunto. A partir do atestado de óbito foram coletadas informações adicionais através de entrevista à família, hospitais, postos de puericultura, ambulatórios e serviços de urgência. Todas as informações registradas foram observadas e os diagnósticos discutidos com os responsáveis pelo atendimento médico. Verificada a realização de necrópsias, os dados foram incluídos visando a análise das causas de óbito. Nos óbitos sem assistência médica, as famílias foram entrevistadas por médico, procurando-se obter a história clínica para tentativa de diagnóstico. Nos demais casos, a entrevista à família foi realizada por visitadora social. De posse de todas as informações existentes, os casos eram submetidos à análise pela equipe responsável pela investigação, procurando-se definir a causa básica do óbito. Para algumas famílias, não localizadas, a investigação limitou-se aos dados fornecidos pelos serviços de saúde. Paralelamente ao estudo dos óbitos realizado no período de julho de 1968 a junho de 1970, foram coletadas informações sobre os nascimentos, diretamente nos serviços obstétricos e confrontadas com as obtidas nos cartórios de registro civil. Após exclusão dos não residentes na área, estes dados permitiram o cálculo do coeficiente de mortalidade infantil. O cálculo do número de menores de 5 anos foi obtido tomando-se $11,7 \%$ da população da área, estimada a partir dos dados de recenseamento. Essa proporção foi a assinalada para o interior do Estado de São Paulo pelo IBGE ${ }^{3}$ (1970). Desta forma foi possí- 
TERUEL, J. R. et al. - Causas de mortalldade na infancia, regiăo de Ribeirăo Preto, São Paulo, Brasil. Rev. Saúde públ., S. Paulo, 7: 67-72, 1973.

vel calcular o coeficiente de mortalidade em menores de 5 anos.

\section{RESULTADOS E DISCUSSAO}

Foram incluídos no estudo 1124 óbitos com a seguinte distribuição: 462 ocorridos entre crianças residentes em Ribeirão Preto, 433 em Franca e 229 nas cinco comunidades. Em 87 casos a família não foi localizada, sendo que 33 delas por motivo de mudança após o óbito da criança.

A Tabela 1 apresenta os dados dos óbi- tos segundo o sexo e grupos etários para o período total de estudo. Em termos absolutos, cerca de metade dos óbitos pertencem ao grupo neonatal. $O$ sexo masculino contribuiu com um maior número de óbitos do que o feminino, respectivamente: 610 e 514 óbitos. Este predomínio do sexo masculino é mais evidente nos 6 primeiros meses de vida.

No periodo da investigação foram constatados 18.248 nascidos vivos de mulheres residentes na área com a seguinte distribuição: Ribeirão Preto: 9314; Franca: 5093 e nas cinco comunidades restantes

T A B E L A 1

Óbitos de menores de 5 anos segundo sexo e grupos estários - Região de Ribeirão Preto (zonas urbanas) - julho de 1968 a junho de 1970

\begin{tabular}{|c|c|c|c|c|c|c|c|}
\hline \multirow{2}{*}{ Sexo } & \multicolumn{7}{|c|}{ Grupos Etários } \\
\hline & $01-28 d$ & $28 \mathrm{~d} \mid-3 \mathrm{~m}$ & $3 \mathrm{~m} \mid-6 \mathrm{~m}$ & $6 \mathrm{~m}-12 \mathrm{~m}$ & $1 \mathrm{a} \mid-2 \mathrm{a}$ & $2 a \mid-5 a$ & $\begin{array}{c}\text { Total } \\
5 \mathrm{a}\end{array}$ \\
\hline Masculino & 290 & 93 & 94 & 57 & 33 & 43 & 610 \\
\hline Feminino & 220 & 77 & 68 & 58 & 46 & 45 & 514 \\
\hline Total & 510 & 170 & 162 & 115 & 79 & 88 & 1124 \\
\hline
\end{tabular}

T A B E I A 2

Coeficientes de mortalidade em menores de 5 anos, coeficientes de mortalidade infantil e coeficientes de mortalidade neonatal - Zonas urbanas de cidades da região de Ribeiräo Preto - junho de 1968 a junho de 1970

\begin{tabular}{|c|c|c|c|c|}
\hline \multirow[b]{2}{*}{ Coeficientes } & \multicolumn{4}{|c|}{ Áreas Urbanas } \\
\hline & $\begin{array}{c}\text { Ribeirão } \\
\text { Preto }\end{array}$ & Franca & $\begin{array}{c}\text { Cinco } \\
\text { Comunidades }\end{array}$ & Total \\
\hline $\begin{array}{l}\text { Mortalidade em menores } \\
\text { de } 5 \text { anos (por } 1000 \text { ha- } \\
\text { bitantes } 5 \text { anos) }\end{array}$ & 10,84 & 22,44 & 14,54 & 14,47 \\
\hline $\begin{array}{l}\text { Mortalidade infantil - } \\
\text { (por } 100 \text { nascidos vivos) }\end{array}$ & 42,84 & 71,27 & 50,77 & 52,44 \\
\hline $\begin{array}{l}\text { Mortalidade neonatal - } \\
\text { (por } 1000 \text { nascidos vivos) }\end{array}$ & 23,51 & 36,52 & 27,34 & 27,95 \\
\hline
\end{tabular}


TERUEL, J. R. et al. - Causas de mortalidade na infâncla, região de Ribeirão Preto, São Paulo, Brasil. Rev. Saúde públ., S. Paulo, 7: 67-72, 1973.

3841. Com estes dados e com a estimativa da população de menores de 5 anos foram calculados os coeficientes de mortalidade apresentados na Tabela 2. Não foram publicados, até o momento, valores de coeficientes de mortalidade, da região, com as correçōes realizadas neste estudo. Acreditamos que os valores apresentados são os que mais se aproximam dos valores reais. A área de Ribeirão Preto apresenta a melhor situação diante dos valores dos coeficientes ao passo que estes, em Franca, atingem niveis mais elevados.

As causas básicas dos óbitos foram codificadas segundo a 8. ${ }^{a}$ Revisão da Classificação Estatística Internacional de Doença, Lesões e Causas de Morte. A Tabela 3 apresenta o resultado da codificação segundo os 17 grupos principais da referida Classificação. Observa-se que apenas dois grupos respondem por mais da metade dos óbitos: Grupo I - Doenças Infecciosas e Parasitárias e Grupo XV -

T A B E L A 3

Obitos de menores de 5 anos segundo grupos de causas - Zona urbana de cidades da região de Ribeirão Preto - julho de 1968 a junho de 1970

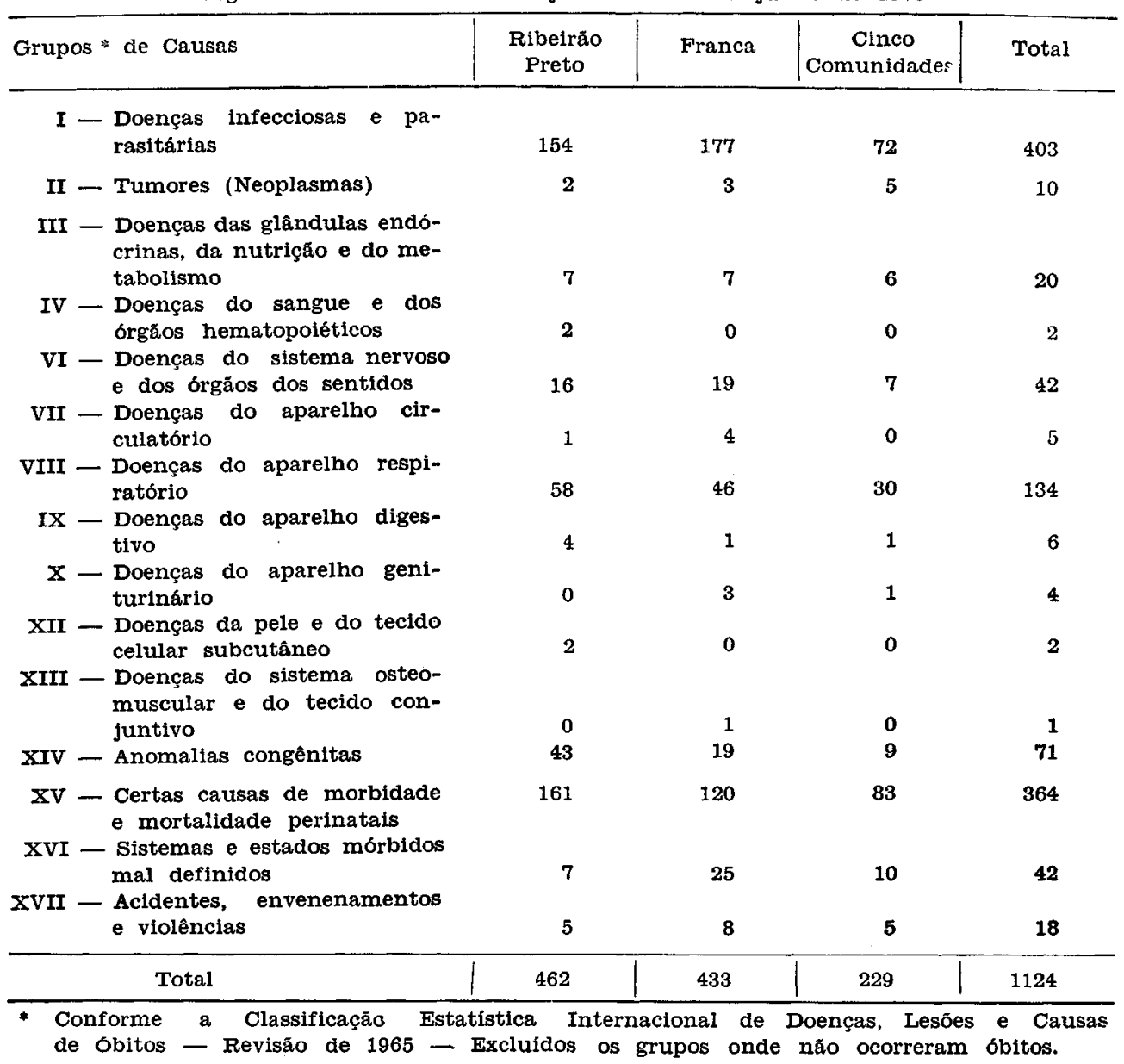


TERUEL, J. R. et al. - Causas de mortaliđade na infância, região de Ribeirão Preto, Săo Paulo, Brasil. Rev. Saúde públ., S. Paulo, 7: 67..72, 1973.

T A B E L A 4

Proporção de óbitos para os principais grupos de causas em menores de 5 anos - Regiāo de Ribeirão Preto - julho de 1968 a junho de 1970

\begin{tabular}{|c|c|c|c|c|}
\hline Grupos * de Causas & $\begin{array}{c}\text { Ribeirão } \\
\text { Preto }\end{array}$ & Franca & $\begin{array}{c}\text { Cinco } \\
\text { Comunidades }\end{array}$ & Total \\
\hline $\begin{array}{l}\text { I - Doenças infecciosas e para- } \\
\text { sitárias }\end{array}$ & $33,3 \%$ & $40,1 \%$ & $31,4 \%$ & $35,9 \%$ \\
\hline $\begin{array}{c}\text { XV - Certas causas de morbidade } \\
\text { e mortalidade perinatais }\end{array}$ & $34,8 \%$ & $27,7 \%$ & $36,2 \%$ & $32,4 \%$ \\
\hline $\begin{array}{l}\text { VIII - Doenças do aparelho respi- } \\
\text { ratório }\end{array}$ & $12,5 \%$ & $10,6 \%$ & $13,1 \%$ & $11,9 \%$ \\
\hline XIV - Anomalias congênitas & $9,3 \%$ & $4,4 \%$ & $3,9 \%$ & $6,3 \%$ \\
\hline Total & $89,9 \%$ & $82,8 \%$ & $84,6 \%$ & $86,5 \%$ \\
\hline
\end{tabular}

* Conforme a Classificação Estatística Intenacional de Doenças, Lesões e Causas de Obitos Revisão de 1965.

Causas Perinatais. Na Tabela 4, estão assinalados os principais grupos de causas responsáveis por cerca de $86 \%$ dos óbitos.

Quanto às causas específicas dos óbitos, que serão objeto de publicação posterior, destacamos a grande frequiência da doença diarréica no grupo das doenças infecciosas, ocorrendo em cerca de $30 \%$ do total de óbitos. A prematuridade e a desnutrição foram importantes causas contributórias.

Certo número de óbitos $(3,7 \%)$ não pôde ser codificado por falta de informações clínicas precisas. Isto ocorreu em $1,5 \%$ dos casos em Ribeirão Preto, 5,8\% dos óbitos ocorridos em Franca e 4,4\% dos pertencentes às cinco comunidades. Estes dados refletem, de certa maneira, diferenças nos recursos assistenciais à infância utilizados pelas famílias, nas áreas estudadas. Parte considerável das crianças residentes nas cinco comunidades obtiveram assistência em Ribeirão Preto onde existe maior concentração de recursos para atenção médica, o que explica a menor proporção de causas mal definidas, nestas comunidades, quando comparadas com a proporção assinalada para a zona urbana de Franca. De certo modo, os valores dos coeficientes de mortalidade apresentados também refletem os problemas de assistência médica, ao lado dos inúmeros fatores sócio-econômicos que influem nos problemas de saúde em diferentes áreas.

Durante o desenvolvimento da Investigação várias observações permitiram evidenciar problemas de atenção médica.

Em diversas oportunidades as mães procuraram mais de um serviço e o atendimento realizado não foi acompanhado da orientação devida. Casos de diarréia em fase inicial evoluíram para formas graves com um mínimo de atuação por parte do pessoal de saúde. $O$ pessoal paramédico é praticamente inexistente. $\mathrm{Em}$ geral se reduz a poucas enfermeiras au- 
TERUEL, J. R. et al. - Causas de mortalidade na infância, regiăo de Ribeirăo Preto, São Paulo, Brasil. Rev. Saúde públ., S. Paulo, 7: 67-72, 1973.

xiliares, em serviços nos hospitais, dando atendimento aos casos mais graves. Torna-se necessário um melhor estudo dos recursos humanos e assistenciais pa- ra a saúde visando uma melhor estrutura de atenção médica e consequientemente melhores níveis de saúde das populações.

\section{RSPU-B/161}

Teruel, J. R. et al. - [Infant mortality causes, County of Ribeirão Preto, S.P., Brazil]. Rev. Saúde públ., S. Paulo, 7:67-72, 1973.

SumMary: The study of 1124 deaths of under 5 years children residing in urban areas in the region of Ribeirāo Preto, Brazil, in the period of June 1968 to July 1970, through the gathering of all information registered at the health care services and obtained through interviews with families and physicions responsible for the caring, is described. The predominance of deaths in the neonatal period is observed. The coefficient of infant mortality was estimated in 52.4 for a thousand live born. The importance of the infectious and perinatal causes is evidenced, as well as the health care problems regarding childhood chiefly and the want of trained paramedical personnel.

UNITERMs: Infant mortality*; Ribeirão Preto, S. Paulo, Brazil"; Infectious diseases.

\section{REFERENCIAS BIBLIOGRAFICAS}

1. ANDRADE, G. S. - Distrofia pluricarencial hidropigênica. J. Pediat., Rio de Janeiro, 19:144-60, 1954.

2. ARAUJo, J. D. - A realidade médico assistencial brasileira. In: REUNIAO ANUAL DA ASSOCIAÇAO BRASILETRA DE ESCOLAS MEDICAS. 7.a, Niterói, 1969. Anais. Rio de Janeiro, 1969. Sub-tema I, p. 27-46.

3. INSTITUTO BRASILEIRO DE GEO. GRAFIA E ESTATISTICA - Pesquisa nacional por amostra de domicilios. São Paulo, 1970. (Doc. GEPD n. 0 42).

4. ORGANIZACION PANAMERICANA DE LA SALUD - Las condiciones de salud. en las Americas: 1965-1968. Washington, D.C., 1970. (Publicación Cientifica n. 207 ).

5. ORganizacion PANAMERICANA DE LA SALUD - Hechos que revelan progresso en salud: metas en la Carta de Punta del Este. Washington, D.C., 1968. p. 58. (Publicactón Clentifica n.० 166).

6. ORGANIZACION PANAMERICANA DE LA SALUD - Investigación In- teramericana de Mortalidad en la Niñez: primer áno de la investigación. Washington, D.C., 1971. p. 160. [Informe provisional].

7. PUfFer, R. R. - Fases iniciales de la Investigación Interamericana de Mortalidad en la Niñez. Bol. Ofic. sanit. panamer., 65:114-26, 1968.

8. REUNIÁO ANUAL DA ASSOCIAÇAO BRASILEIRA DE ESCOLAS MÉDICAS. 7. a, Niteró, 1969. Anais. Rio de Janeiro, 1969. p. 347.

9. SILVA, G. R. da - Formação do médico de acordo com as diferenças regionais e a diversidace das equipes de saúde. In: REUNIAO ANUAL DA ASSOCIAÇAO BRASILEIRA DE ESCOLAS MtDICAS. 7.a, Niterói, 1969. Anais. Rio de Janeiro, 1969. Subtema III, p. 95-128.

10. SIMÖES, A. J. P. - - pessoal de saúde necessário. Ibdem. Sub-tema II, p. 49-68.

Recebido para publicação em 31-1-1973 Aprovado para publicação em 4-4-1973 